\title{
构件不同部位粗楉度对疲劳性能影响差异分析
}

\author{
赵丙峰 ${ }^{1,2}$ 谢里阳 ${ }^{1,2}$ 李 冲 ${ }^{1,2}$ 王博文 ${ }^{1,2}$ 秦 波 ${ }^{1,2}$ \\ (1. 东北大学机械工程与自动化学院 沈阳 110819; \\ 2. 东北大学航空动力装备振动及控制教育部重点实验室 沈阳 110819)
}

\begin{abstract}
摘要: 为验证构件不同部位粗䊅度影响的差异性, 以某型铝合金为研究对象, 采用疲劳试验与理论分析相结合的方法分析不 同部位粗粘度组合下构件疲劳性能的差异。试验设计中, 为区分不同部位粗粘度造成的影响, 着重对试样角部、面部和曲面 部做不同的表面处理, 同时设计棒试样及两类厚度的板试样以说明试验结果的普适性, 并在试验结果处理中通过观测试样的 断口形貌定义面断裂(曲面断裂)与角断裂两种断裂形式。为进一步定量分析不同部位粗粘度影响差异性的大小，采用有限元 软件分析不同部位微缺口附近的应力分布特性, 另外, 针对本研究的特殊性提出一种适用于微尺寸缺口处应力计算的改进场 强法, 并采用此方法分别计算试样表面各类微缺口处的有效破坏应力及有效应力集中系数。通过试验结果与理论计算结果对 比发现, 对于同样表面粗䊚度的试样, 不同部位微缺口处的有效应力集中系数存在显著差异, 即角部微缺口处集中系数明显 高于面部, 这一结论反映到宏观上表现为试样边角处的粗樯度对试样疲劳性能的影响明显高于试样面部粗粘度的影响, 因此 传统方法中将两者做等同处理缺乏一定的合理性。
\end{abstract}

关键词: 铝合金; 粗粘度; 应力集中; 疲劳试验; 场强法

中图分类号: 0346

\section{Analysis on the Different Effect of Roughness in Different Part of Structure on Fatigue Property}

\author{
ZHAO Bingfeng $^{1,2}$ XIE Liyang ${ }^{1,2}$ LI Chong ${ }^{1,2}$ WANG Bowen ${ }^{1,2}$ QIN Bo ${ }^{1,2}$
}

(1. School of Mechanical Engineering and Automation, Northeastern University, Shenyang 110819;

2. Key Laboratory of Vibration and Control of Aeronautical Power Equipment,

Northeastern University, Shenyang 110819)

\begin{abstract}
In order to verify the difference effect of roughness in different part of structure, taking a certain type of aluminum alloy as the research object, fatigue test and theoretical analysis are used to analyze the difference of fatigue performance under the combination of different roughness. In the test, emphasis is placed on different surface treatment combinations for corner, face and curved face to find the difference between the effects of the roughness of different parts. In addition, bar specimens and two kinds of thickness plate specimens are designed to illustrate the universality of the test results and two fracture forms of plane fracture (surface fracture) and angle fracture are defined by observing the fracture surface of the specimens In the test results processing. Finite element software is used to analyze the stress distribution characteristics near the micro notch of different parts to quantitatively analyze the difference of the roughness of them. For the particularity of this study, an improved field strength method for stress calculation of micro notch is proposed and effective failure stress and effective stress concentration factor at various micro notches on the specimen surface are calculated by this method. By comparing the experimental results with the theoretical results, it is found that there are significant differences in effective stress concentration coefficient at different locations, which means the concentration coefficient at the corner microgap is obviously higher than that of the face, for the sample with the same surface roughness. This conclusion is reflected in the macroscopic view that the roughness at the corner of the specimen has a greater effect on the fatigue performance of the specimen than the roughness of the specimen surface, which means it is not reasonable to treat them equally in traditional way.
\end{abstract}

Key words: aluminum alloy; roughness; stress concentration; fatigue test; field intensity method

* 国家自然科学基金资助项目(U1708255)。20190117 收到初稿, 20190715 收到修改稿 


\section{0 前言}

表面粗粘度是指材料表面上的较小间距和峰谷 所组成的微观几何形状特征, 是反映构件表面微观几 何形貌的一个重要指标, 其对构件疲劳性能有非常显 著的影响 ${ }^{[1-2]}$ 。断裂力学研究表明, 材料表面粗䊁度值 越大, 其表面的沟痕越深、纹底半径越小，从而表面 应力集中越严重。在交互载荷作用下，应力集中能加 速疲劳裂纹的形核生长, 从而加剧构件的疲劳破坏 ${ }^{[3]}$ 。 因此, 表面粗粘度值增大会降低构件的疲劳强度, 这一现象在实际工程应用中应引起广泛的关注。

对研究表面粗䊁度对构件疲劳寿命影响程度这 一问题, 分析最早、应用也较为广泛的一种思路即为 直接探求构件表面粗糙度与其疲劳寿命的关系, 因为 研究较早, 其方法也较为多样 ${ }^{[4]}$ 。刘军等 ${ }^{[5]}$ 、SILVA 等 ${ }^{[6]}$ 和 WANG 等 ${ }^{[7]}$ 都在相关领域做了大量试验, 试图 通过最终的试验直接寻求不同表面粗糙度下构件疲 劳寿命的变化规律, 并建立了相应的预测模型。

HATAMLEH 等 ${ }^{[8]}$ 研究了激光喷丸、喷丸硬化及两者 结合对摩擦摚拌焊接部位疲劳寿命的影响, 而区分 这三种表面处理技术的表征参数即为表面粗粘度。

与 HATAMLEH 的研究类似, BAGEHORN 等 ${ }^{[9]}$ 研究 了 Ti-6Al-4V 材料在激光束切割、锻压等加工工艺 下不同表面粗粘度对板材与构件接头疲劳性能的影 响。以上两者研究结果都表明, 不同加工方式下表 面粗粘度对构件疲劳性能的影响是存在差异的, 但 整体趋势都表现为表面粗粘度越高, 构件疲劳性能 越好。与以上思路不同, LI 等 ${ }^{[10]}$ 另辟蹊径, 采用平 均表面粗粘度的概念来研究材料的疲劳问题, 并以 此为基础针对不同表面粗楉度的中碳钢, 提出了建 立材料 $S-N$ 和 $P-S-N$ 曲线的新方法。以上的直接分 析方法可简单通过试验进行总结分析, 其计算过程 明确, 模型也较为简洁, 因此在工程上应用较为广 泛。但近年来, 基于相关研究及工程实践总结的大 量经验, 有学者 ${ }^{[11-12]}$ 指出直接采用构件表面微观几 何参数表征疲劳寿命是不合理的, 建议先在微观层 面上对构件表面进行应力集中分析, 然后基于应力 分析结果间接预测构件疲劳寿命。但因粗粘度所处 的量级上材料表面形貌分布复杂, 直接基于试样原 始微观形貌来分析其缺口处的应力分布很难实现, 对此, 有学者在仿真前对表面微观形貌进行简化处 理, 然后以此基础上建立有限元模型进行应力分 析 ${ }^{[13-14]}$ 。目前, 针对表面微观形貌的简化处理方法有 很多, 其中以下面几种较为常见。ANDREWS 等 ${ }^{[15-17]}$ 提出将表面微缺口形貌简化为半粗圆形缺口, 进而
在此简化的基础上采用计算机仿真软件进行模拟， 这也是现如今较为常用的简化方法。另外, AROLA 等 ${ }^{[18]}$ 和 EMBRECHTS ${ }^{[19]}$ 为评估高强度钢表面的应 力集中状态，将材料表面的复杂形貌简化成理想的 正弦状微缺口, 提出了 Arola-Ramulu 模型, 并通过 AISI 4130 CR 钢的试验数据将其与 Neuber 公式进 行了对比。程正坤等 ${ }^{[20]}$ 基于线弹性力学理论, 采用 谐波叠加的方式模拟材料表面形貌，提出了一种新 的表面形貌有限元建模方法，该方法能反映机加工 表面的总体应力集中水平，具有较高的工程应用价 值。基于以上有限元分析求解过程，可列出构件表 面微缺口附近的应力分布, 但以上应力分析的主要 目的是获得构件的疲劳性能, 因此这两者之间需有 一个有效的纽带模型进行联结。对此, SURARATCHAI 等 ${ }^{[21]}$ 和 ALANG 等 ${ }^{[22]}$ 对不同表面 粗粘度的试样进行了四点弯曲疲劳测试, 并结合有 限元法给出了不同表面应力集中下的材料的 $S-N$ 曲 线及疲劳极限分布, 并将试验结果与理论计算结果 进行了对比。另外, ALENCAR 等 ${ }^{[23]}$ 和 GAUR 等 ${ }^{[24]}$ 也都研究了基于有限元分析结果的疲劳寿命预测问 题, 这一系列成果在本领域的诸多研究中 ${ }^{[25-26]}$ 都有 广泛应用, 也为解决本文中的问题提供了参考。

以上理论在分析表面粗楉度对构件疲劳性能的 影响时, 大多选取构件表面粗粘度进行研究, 即忽 略了构件面部粗楉度的影响。但从试验上来看, 对 于圆柱形等标距段截面为中心对称图形的试样, 这 一简化方式是没有异议的。但对于板形及其他标距 段截面存在长轴或短轴的试样, 研究 ${ }^{[5,21,27]}$ 仍只以 试样面部微缺口处应力分析为基础进行构件瘦劳寿 命估算, 这就意味着在疲劳寿命与构件表面粗粘度 关系的研究中, 主观地忽略了构件角部粗粘度对其 疲劳性能影响的特殊性, 而默认了不同部位粗糙度 对构件疲劳性能的影响是等同的，这一简化过程明 显缺乏理论支撑。为验证这一假设是否合理, 本文 以某型铝合金板试样、棒试样为研究对象, 对试样 不同部位进行了表面处理，通过高周疲劳试验，获 得各组试样疲劳极限。另外, 为定量分析不同部位 粗䊁度对构件疲劳性能影响的差异性, 采用有限元 软件分析了不同部位微缺口附近的应力分布特性, 并采用改进后的场强法计算了各类微缺口处的有效 破坏应力及有效应力集中系数。通过分析结果对比 发现，对于同样表面粗糙度的构件，不同部位微缺 口处的有效应力集中系数存在显著差异, 这一结论 反映到宏观上表现为试样边角处的粗粘度对试样疲 劳性能的影响明显高于试样面部粗糙度的影响, 因 此将两者做等同处理缺乏一定的合理性。 
1 各类角、面粗糙度组合下的材料疲 劳极限测试

\section{1 试验规划}

本次试验所有过程均参照 GB/T3075 标准, 试 样材料为铝合金, 分板试样、棒试样两种规格, 基 本尺寸见图 1。图 1 中, 中间 $45 \mathrm{~mm} 、 20 \mathrm{~mm}$ 长
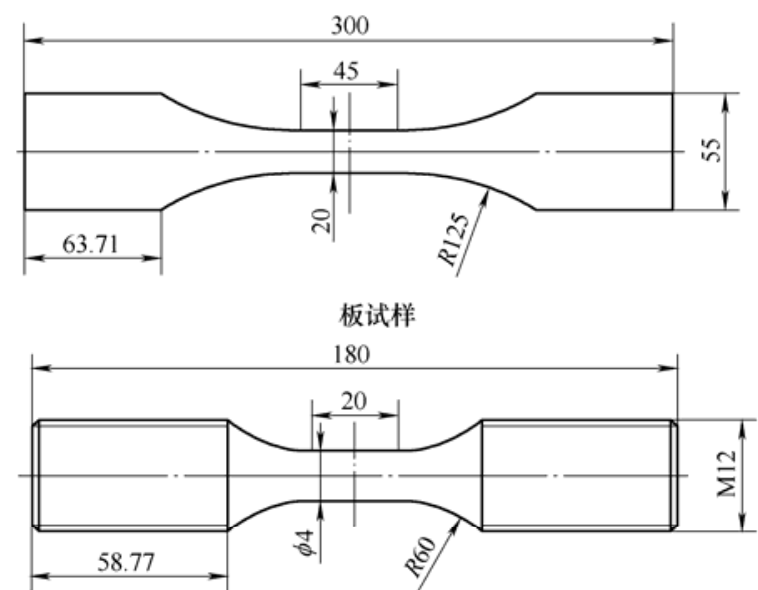

棒试样

(a) 试样二维图 $(\mathrm{mm})$

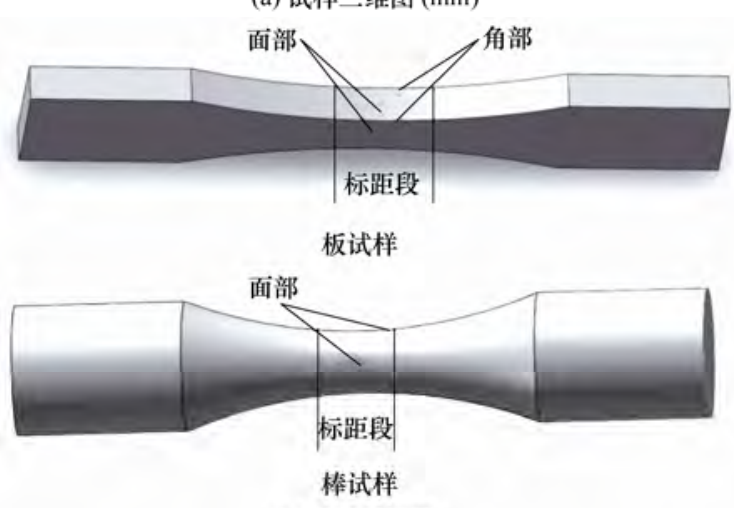

(b) 试样三维图

图 1 试样基本尺寸及形貌
度处为试样标距段。为考虑厚度及板型、表面形貌 对试样疲劳寿命的影响, 试验共设计了八类试样 (表 1)进行对照, 且每类试样需至少获取 15 个有效 数字。另外, 因为打磨面部时不可避免的会对角部 表面质量造成影响，因此针对板试样并未设置只打 磨面部而角部不作处理的对照组。

\section{表 1 试样种类列表}

\begin{tabular}{|c|c|c|c|c|}
\hline 试样规格 & $\begin{array}{c}\text { 试样标距段 } \\
\text { 处理方式 }\end{array}$ & $\begin{array}{c}\text { 面部粗粘度/ } \\
\mu \mathrm{m}\end{array}$ & $\begin{array}{c}\text { 角部粗徙度/ } \\
\mu \mathrm{m}\end{array}$ & $\begin{array}{l}\text { 试样 } \\
\text { 代号 }\end{array}$ \\
\hline \multirow{3}{*}{$\begin{array}{c}\text { 厚度 } 4 \text { mm } \\
\text { 板材 }\end{array}$} & 未打磨 & 1.6 & 1.6 & A-1 \\
\hline & 打磨角部 & 1.6 & 0.4 & A-2 \\
\hline & 全打磨 & 0.4 & 0.4 & A-3 \\
\hline \multirow{3}{*}{$\begin{array}{c}\text { 厚度 } 10 \mathrm{~mm} \\
\text { 板材 }\end{array}$} & 未打磨 & 1.6 & 1.6 & B-1 \\
\hline & 打磨角部 & 1.6 & 0.4 & B-2 \\
\hline & 全打磨 & 0.4 & 0.4 & B-3 \\
\hline 直径 4 mm & 未打磨 & 1.6 & 1.6 & C-1 \\
\hline 棒材 & 全打磨 & 0.4 & 0.4 & $\mathrm{C}-2$ \\
\hline
\end{tabular}

本次疲劳试验采用电磁谐振式高频疲劳试验 机(QBG200), 采用轴向力正弦波加载的方式, 应 力比 $R$ 为 0 , 加载频率在 $150 \mathrm{~Hz}$ 与 $180 \mathrm{~Hz}$ 之间。 疲劳极限测试试验方法采用升降法 ${ }^{[28]}$, 应力起始 值为 $190 \mathrm{MPa}$, 应力级差取 $8 \mathrm{MPa}$, 数据处理选 用配对法 ${ }^{[29]}$ 。另外, 本试验规定寿命为 $1.0 \times 10^{7}$ 应力循环次所对应的应力级为该试样的疲劳极 限, 试样在规定应力下, 一直持续试验至试样失 效或规定循环次数(溢出)。试样失效应发生在试 样标距段(试样的最大应力截面), 否则试验结果 无效。

\section{2 试验结果}

按照以上试验规划, 得到的试验结果见表 2 。 其中, 表中只列出了试验的有效数据, 应力级单 位为 $\mathrm{MPa}$ 。另外, 为区分不同的试验事件, “+” 表示试样结果为溢出, “-”表示试样结果为断裂。

表 2 试验结果列表

\begin{tabular}{|c|c|c|c|c|c|c|c|c|}
\hline 应力等级编号 & A-1 & A-2 & A-3 & B-1 & B-2 & B-3 & C-1 & $\mathrm{C}-2$ \\
\hline 1 & $190 /-$ & $190 /+$ & $190 /+$ & $190 /-$ & $190 /+$ & $190 /+$ & $190 /+$ & $190 /+$ \\
\hline 2 & $182 /+$ & $198 /+$ & 198/+ & $182 /-$ & 198/- & $198 /+$ & $198 /-$ & $198 /+$ \\
\hline 3 & $190 /-$ & $206 /-$ & $206 /+$ & $174 /-$ & $190 /+$ & $206 /+$ & $190 /-$ & $206 /+$ \\
\hline 4 & $182 /-$ & $198 /+$ & $214 /-$ & $166 /+$ & $198 /-$ & $214 /+$ & $182 /+$ & $214 /+$ \\
\hline 5 & $174 /-$ & $206 /+$ & $206 /+$ & $174 /+$ & $190 /+$ & $222 /+$ & $190 /+$ & $222 /-$ \\
\hline 6 & $166 /+$ & $214 /+$ & $214 /+$ & $182 /-$ & $198 /+$ & $230 /+$ & $198 /+$ & $214 /+$ \\
\hline 7 & $174 /-$ & $222 /-$ & $222 /-$ & $174 /-$ & $206 /-$ & $238 /-$ & $206 /-$ & $222 /+$ \\
\hline 8 & $166 /+$ & $214 /-$ & $214 /+$ & $166 /+$ & $198 /+$ & $230 /-$ & $198 /-$ & $230 /+$ \\
\hline 9 & $174 /-$ & $206 /+$ & $222 /-$ & $174 /-$ & $206 /-$ & $222 /-$ & $190 /-$ & $238 /-$ \\
\hline 10 & $166 /+$ & $214 /+$ & $214 /-$ & $166 /+$ & $198 /+$ & $214 /-$ & $182 /-$ & $230 /-$ \\
\hline 11 & $174 /+$ & $222 /-$ & $206 /+$ & $174 /+$ & $206 /+$ & $206 /+$ & $174 /-$ & $222 /-$ \\
\hline 12 & $182 /+$ & $214 /+$ & $214 /+$ & $182 /-$ & $214 /+$ & $214 /-$ & $166 /+$ & $214 /-$ \\
\hline 13 & $190 /+$ & $222 /-$ & $222 /+$ & $174 /-$ & $222 /+$ & $206 /+$ & $174 /+$ & $206 /+$ \\
\hline 14 & $198 /-$ & $214 /-$ & $230 /+$ & $166 /-$ & $230 /+$ & $214 /-$ & $182 /+$ & $214 /+$ \\
\hline 15 & $190 /-$ & $206 /-$ & $238 /-$ & $158 /+$ & $238 /-$ & $206 /-$ & $190 /+$ & $222 /+$ \\
\hline
\end{tabular}


通过配对法计算, 分别求出八类试样疲劳极限 均值及标准差, 见图 2。由图 2 可得, 对于板试样 和棒试样两类试样, 试样表面质量对其疲劳极限大 小均有显著影响, 且试样表面质量越高其疲劳极限 越高, 这一趋势与其他学者得到的结论是吻合的。 然而, 通过对比未打磨试样数据和打磨角试样数据 以及未打磨试样数据和全打磨试样数据可以发现, 板试样角部表面质量对试样疲劳极限的影响明显高 于面部, 而对于棒试样上的曲面质量对试样疲劳极 限的影响程度界于以上两者之间, 这与传统方法中 的不同部位粗䊁度影响等同性假设是矛盾的。

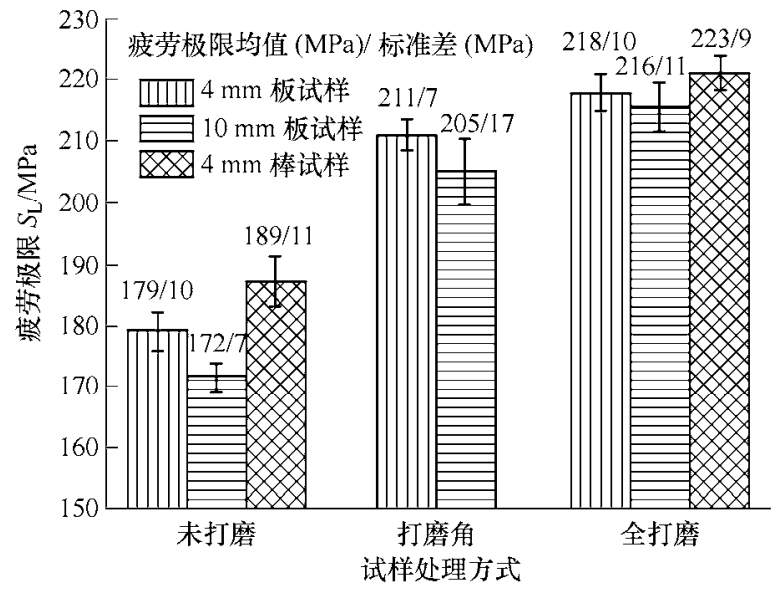

图 2 六类试样疲劳极限均值及标准差

\section{3 测试结果分析}

通过观察试样断口形貌发现, 对于棒试样, 其 疲劳裂纹都起源于试样曲形表面上; 而对于板试样, 其疲劳裂纹可能起源于试样的两个部位(面部和角 部), 对此需要引用疲劳断裂的两个概念: 面断裂(对 于棒试样表现为曲面断裂)和角断裂。面断裂是指断 裂主裂纹萌生于试样面部(曲面断裂则对应试样曲 面部), 主要受试样面部应力集中的影响, 其断口形 貌如图 3a 所示; 角断裂是指断裂主裂纹萌生于试样 角部, 主要受试样角部应力集中的影响, 其断口形 貌如图 3b 所示。为更直观地展示试样断裂形式与试 样粗粘度及加载环境的关系, 结合两种断裂形式, 对以上不同厚度板试样试验结果进行面断裂、角断 裂和溢出的分布统计，做出升降图如图 4 所示。

通过以上分析可以发现, 对于矩形截面试样(板 试样), 无论厚度为 $4 \mathrm{~mm}$ 还是 $10 \mathrm{~mm}$, 其疲劳失效 类别中角断裂均占主体，但厚试样出现面断裂的概 率明显高于薄试样。这主要是因为在其他条件不变 的情况下, 试样厚度越厚, 试样面部所占的比例越 大，因此造成裂纹萌生的集中力也更易出现在面部。 另外, 结合不同的打磨试样, 进一步分析试验结果 可得以下结论。

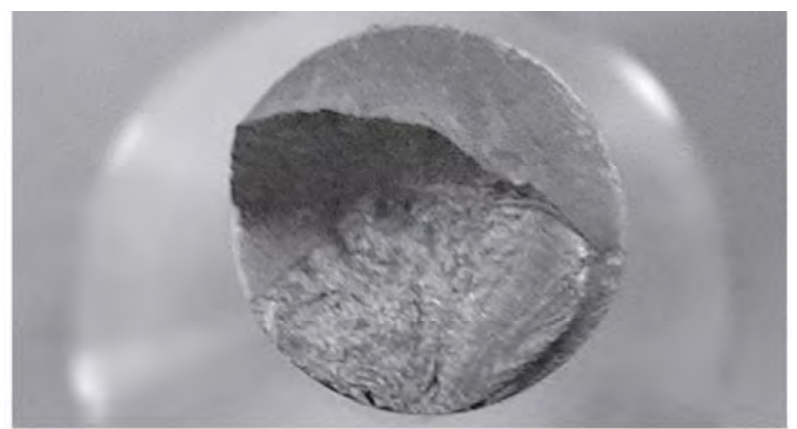

曲面断口宏观形貌

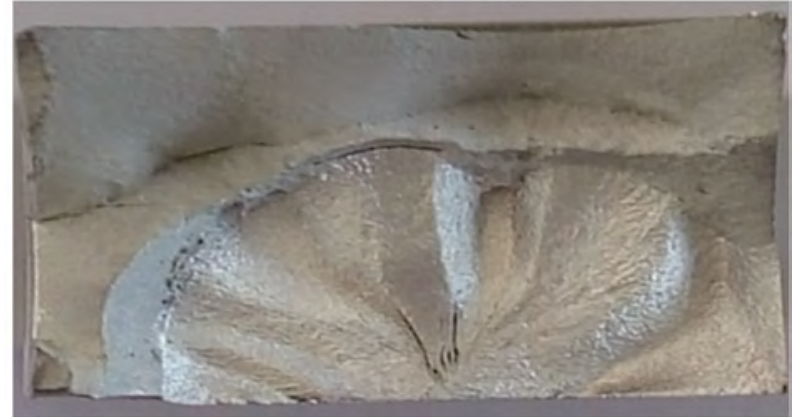

面断口宏观形貌

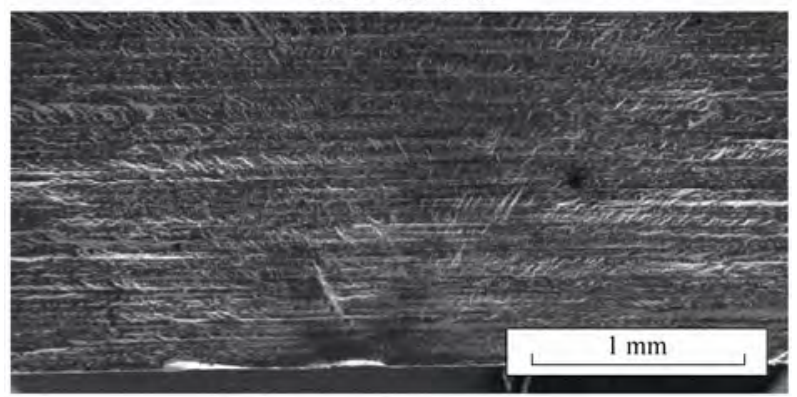

面断口微观形貌 (放大 100 倍)

(a) 曲面 / 面断裂断口形貌

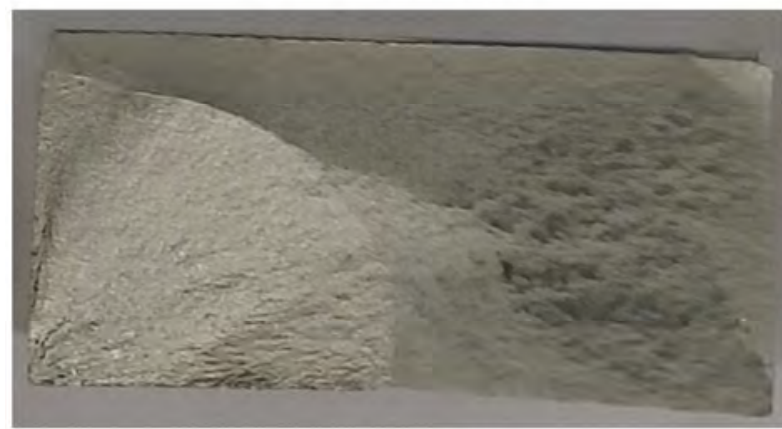

角断口宏观形貌

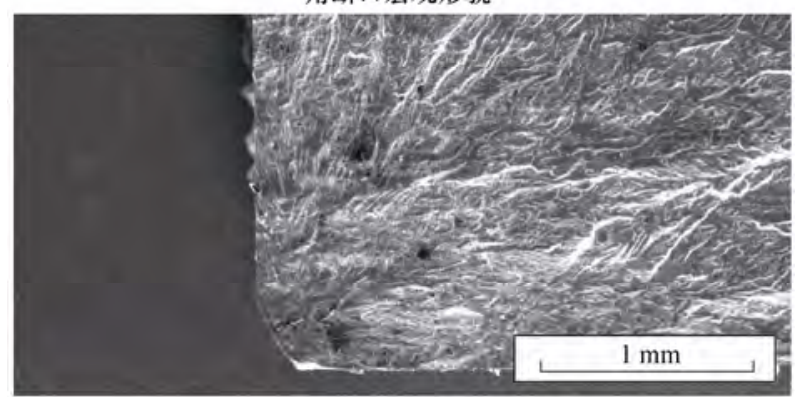

角断口微观形貌 (放大 100 倍)

(b) 角断裂断口形貌

图 3 不同断裂形式的断口宏观/微观形貌 


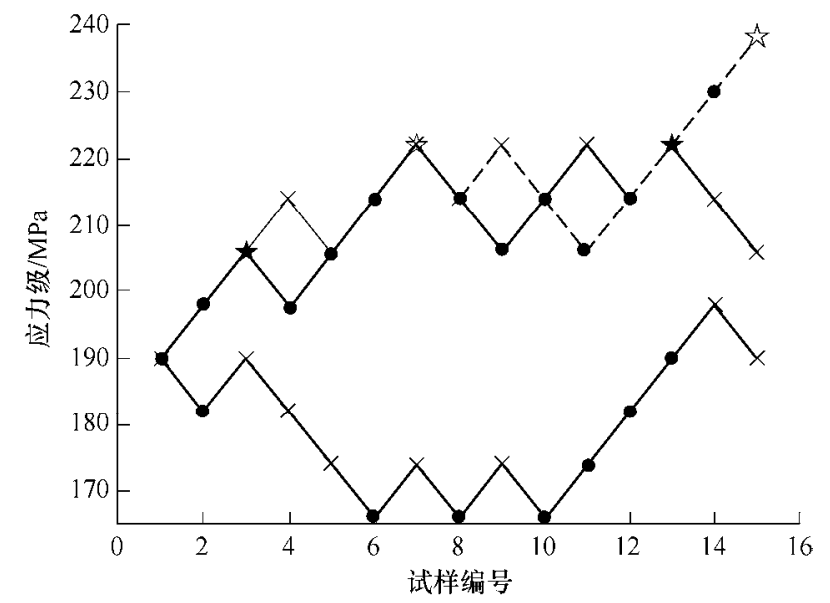

(a) 厚度 $4 \mathrm{~mm}$

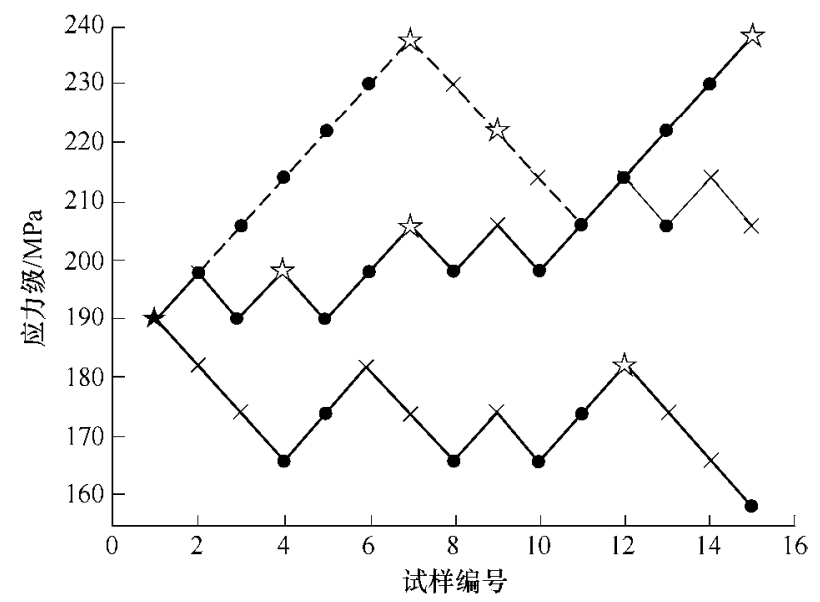

(b) 厚度 $10 \mathrm{~mm}$

$$
\text { 一来打磨 }--- \text { 打磨角 }- \text { 全打磨 }
$$

图 4 不同厚度板试样试验结果升降图

（1）仅打磨角部能使试样破坏由原来的角断裂 向面断裂转换, 打磨面部后又会使角断裂的数量增 加。即角部表面质量的提高能增加面断裂出现的概 率, 面部表面质量的提高能减小面断裂出现的概率。

（2）在其他条件都相同的条件下，试样面断裂 所处的应力级总体高于角断裂所处的应力级, 即同 样外部条件下试样面断裂占总断裂数的比例越高, 其疲劳极限越高。

以上两点整体可概括为, 试样角部表面质量 对试样疲劳极限的影响更为显著, 即试样边角处 粗䊁度与面部粗粘度对材料疲劳极限的影响不是 等同的。

通过上面分析可以发现, 试样边角处粗粘度与 面部粗粘度影响的非等同性很大程度上表现在面断 裂与角断裂的差异性上。为进一步分析面断裂与角 断裂的区别, 下面从微观层面上对两者进行描述。 试验选用两台不同的扫描电镜对部分疲劳试样的断 口进行扫描拍照, 分别分析了面断裂和角断裂两种
情况下裂纹源处的微观形貌, 两者对比如图 5、6 所示(放大倍率都为 2000 倍)。通过对比图 5、6 可 发现, 面断裂疲劳源处微观形貌分布比较规律，且 疲劳辉纹特征显著, 为典型的疲劳裂纹扩展过程; 角断裂疲劳源处微观形貌较为杂乱, 疲劳区与脆性 断裂区交叉分布, 并非典型的疲劳裂纹扩展过程。 因此，除去宏观表征的差异，面断裂与角断裂从断 裂机理上也存在显著区别, 这也间接表明试样边角 处粗䊁度与面部粗糙度会引发试样产生不同的破坏 机制。

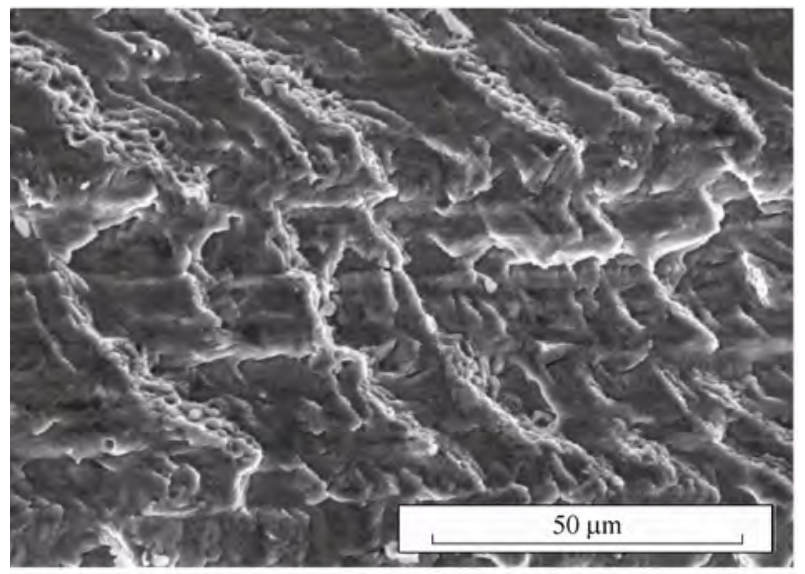

(a) 电镜一

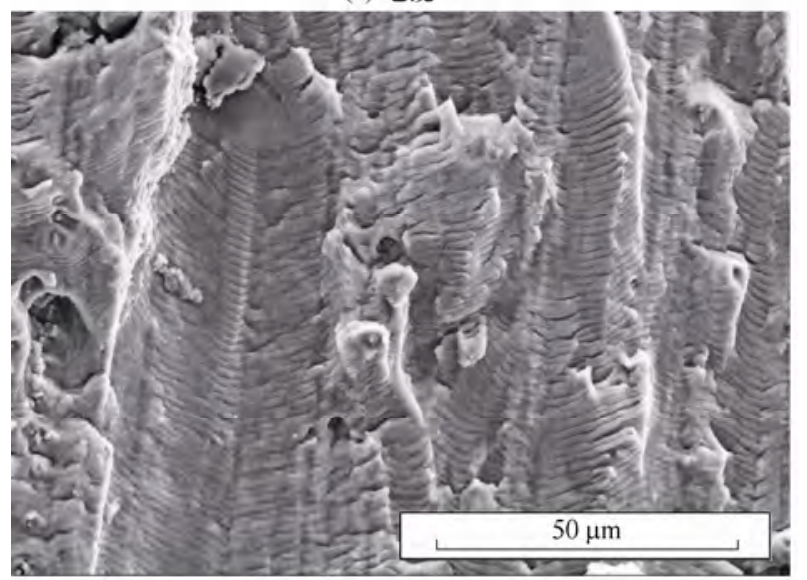

(b) 电镜二

图 5 面断裂疲劳源处微观形貌(放大 2000 倍)

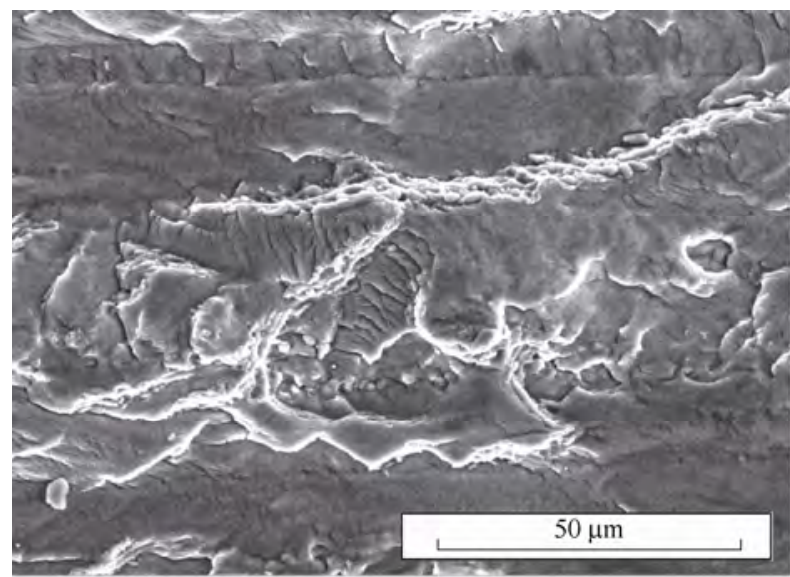

(a) 电镜一 


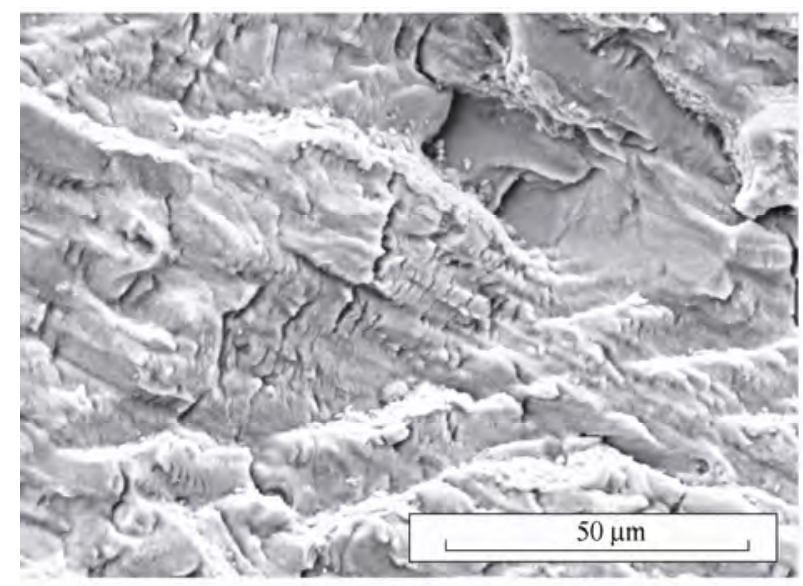

(b) 电镜二

图 6 角断裂疲劳源处微观形貌(放大 2000 倍)

综上所述, 对于板试样, 无论是从宏观表征还是 微观机理上分析, 试样边角处的粗粘度对试样疲劳极 限的影响与试样面部的粗䊁度影响存在显著差异, 且 这一差异在薄厚两种试样中都普遍存在。因此, 在今后 的疲劳问题研究中, 两处的表面粗䊅度必须注意区分。

\section{2 试验结果仿真及理论计算分析}

\section{1 试验结果仿真}

第1.3节仅对试验结果的表观特征进行了总结, 为进一步定量分析不同部位粗粘度对试样疲劳性能 影响的差异性, 本节采用 ABAQUS 分析了不同表 面粗糙度下试样危险部位的应力情况。因试样整体 尺寸与本文提及的粗粘度尺寸 $(R a=0.4 \mu \mathrm{m} 、 1.6 \mu \mathrm{m})$ 存在量级差异, 本节只从试样标距段选取了部分进 行分析, 两种试样选取的分析块如图 8 所示, 长 $\times$ 宽 $\times$ 高 $=100 \mu \mathrm{m} \times 100 \mu \mathrm{m} \times 100 \mu \mathrm{m}$ 。另外, 本文旨 在分析不同部位粗粘度对试样表面应力集中影响差 异性(间接反映两者对疲劳寿命的影响)的定量关系, 因此在有限元分析过程中，在选取分析块上各部位的 微缺陷仅各选取一处 $(m$ 处为面部缺陷, $n$ 处为角部缺 陷, $v$ 为曲面部缺陷), 分别表达为球形微缺口的形式, 如图 7 所示。本文试样分别有 $0.4 、 1.6$ 两类粗鋉度, 因而缺口参数 $h$ 分别相应取 $0.4 \mu \mathrm{m} 、 1.6 \mu \mathrm{m}$ 。分析过 程中, 分析块两段施加均布力, 并可通过下式将两 端施加的力转换为分析块各点所受的名义应力

$$
\sigma=100 F
$$

式中, $\sigma$ 为试样所受名义应力, 单位 $\mathrm{MPa} ; F$ 为仿 真过程中试样两端施加的力, 单位 $\mathrm{N}$ 。

在以上模型假设的基础上, 对模型进行弹塑性 分析, 分析块整体应力云图如图 8a 所示, 从图中可 以看出, $m 、 n$ 两处微缺口处均存在明显的应力集中 现象。为展示两处微缺口附近的应力分布, 分别在 每处微缺口附近选取一条典型的应力分布路径, 如
图 8b 所示。以初始加载应力 $190 \mathrm{MPa}$ 下的板试样 为例, 两种表面粗䊁度下微缺口附近路径应力分布 见图 9。从图 9 可以发现, 加载应力为 $190 \mathrm{MPa}$ 时, 同种表面粗粘度下 $m 、 n$ 两处微缺口处的应力峰值 几乎相同, 这一结果明显与图 4、表 3 中出现角断 裂概率明显大于面断裂这一现象不符。为验证这一 情形的普遍性, 另外分别分析了两类试样在表 2 中 其他应力等级下的微缺口处应力峰值, 并将分析结 果绘制到图 10 中。分析发现, 对于其他应力等级, 上面得到的结论仍然成立，即在相同粗粮度下，同
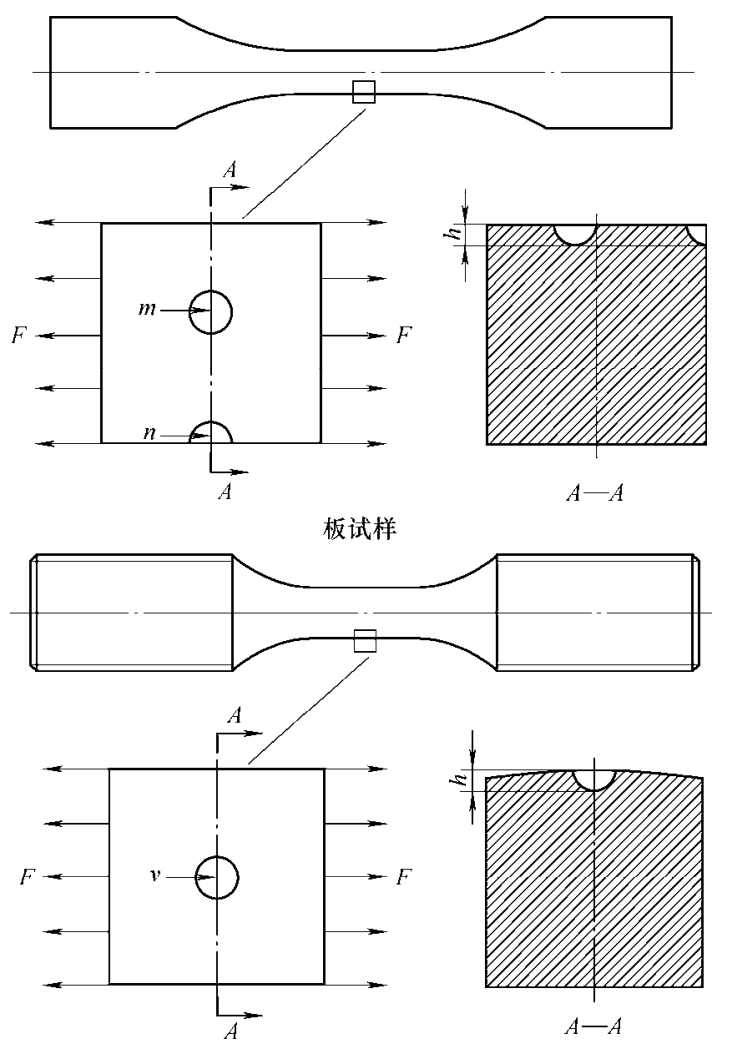

棒试样

图 7 有限元分析单元取样示意图

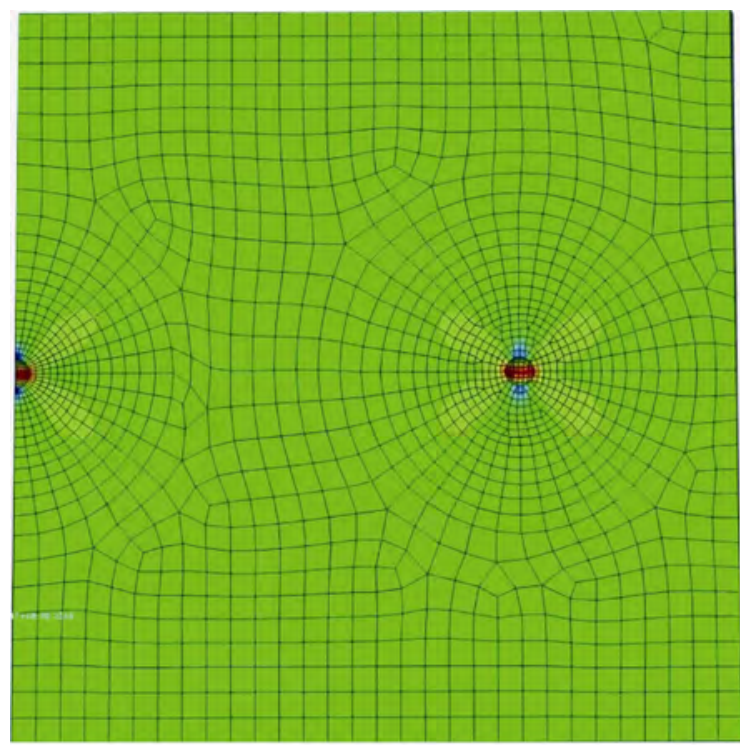

(a) 宏观结构云图 

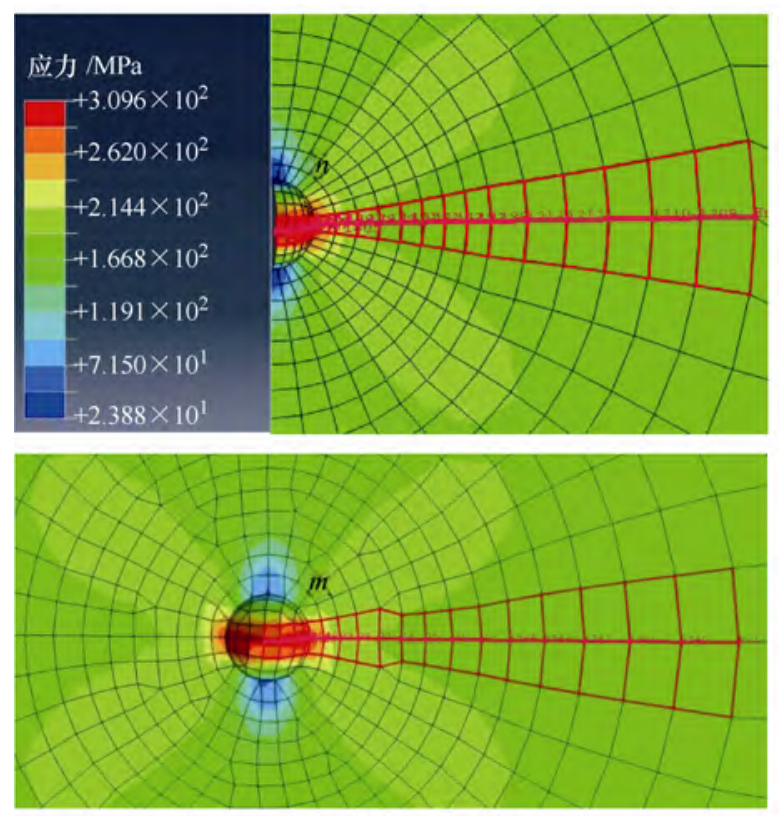

(b) 局部结构云图 (MPa)

图 8 分析块应力云图及微缺口附近路径选取

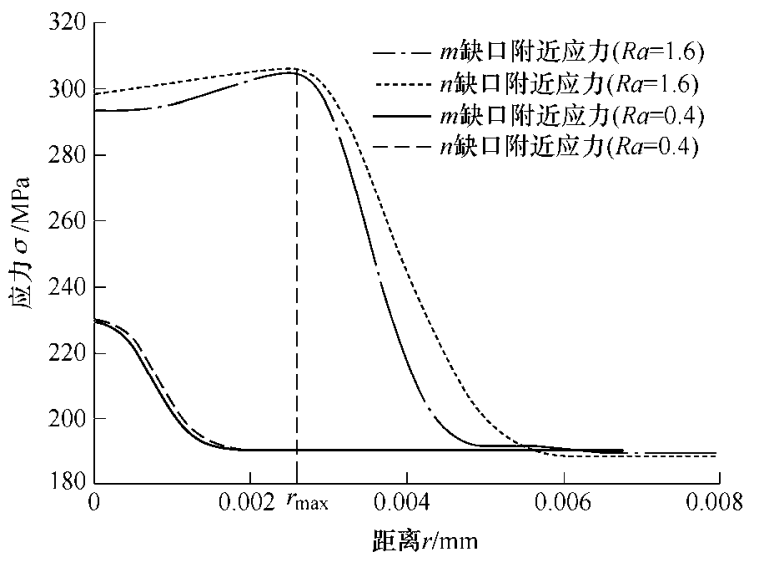

图 9 应力 $190 \mathrm{MPa}$ 时微缺口附近路径应力分布

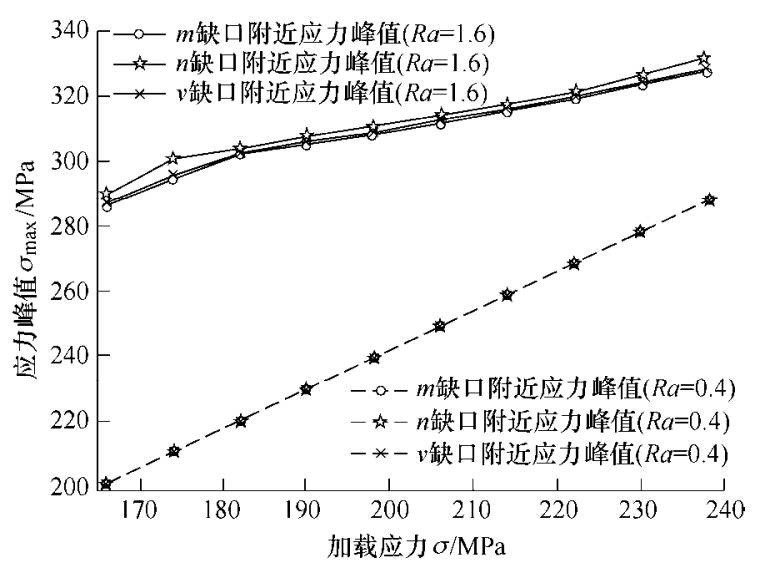

图 10 不同加载应力下微缺口附近应力峰值

一试样 $m 、 n 、 v$ 三处微缺口的应力峰值几乎相同, 且试样表面质量越高, 这一现象越明显。

\section{2 基于改进场强法的微缺口处应力分析}

通过第 2.1 节分析发现, 有限元仿真得到的峰 值结果明显与以上得到的试验结果不符, 因此单靠 微缺口附近的应力峰值解释边角处粗粘度与面部粗
糙度影响的非等同性是行不通的。不仅是在本研究 中, 对于普遍性的疲劳问题, 很多学者都得出结论: 在分析缺口构件疲劳寿命时，仅考虑一 “点” 处的 峰值应力是不够的, 还应在此基础上考虑一定区域 内的应力场 ${ }^{[30]}$ 。基于这一理念, 求解疲劳寿命的应 力场强法 ${ }^{[31-33]}$ 应运而生, 它能考虑缺口效应、尺寸 效应、载荷加载效应及多轴应力效应对疲劳强度及 寿命的影响, 并已得到了多方面的验证。在计算疲 劳损伤的过程中, 场强法充分考虑危险点附近受损 伤区域内材料的应力应变特征, 提出以应力场强 $\sigma_{F I}$ 作为局部区域内疲劳损伤的控制参量, 有

$$
\sigma_{F I}=\frac{1}{V} \int_{\Omega} \varphi(r-\theta) \sigma_{r-\theta} \mathrm{d} v
$$

式中， $\Omega$ 为疲劳破坏区; $V$ 为 $\Omega$ 的体积; $r$ 为参考 点到应力峰值点的距离; $\theta$ 为参考点与缺口对称线 的夹角; $\sigma_{r-\theta}$ 参考点处的应力; $\varphi(r-\theta)$ 为权函数, 常见表达式为

$$
\varphi(r-\theta)=1-\left|\frac{1}{\sigma_{\max }} \frac{\mathrm{d} \sigma_{r-\theta}}{\mathrm{d} r}\right| r(1+\sin \theta)
$$

式中, $\mathrm{d} \sigma / \mathrm{d} r$ 为参考点附近应力梯度; $\sigma_{\max }$ 为缺口 处应力峰值。

在采用传统场强法计算缺口处场强值前, 首先需 要确定 $\Omega$ 的大小, 原场强法认为它只与材料性能有关, 对于疲劳问题, $\Omega$ 一般为数个晶粒的尺寸。但对于粗 楉度的量级来讲, $\Omega$ 的这一定义显然是不合理的, 为 进一步说明这一问题, 同样以初始加载应力 $190 \mathrm{MPa}$ 下的板试样为例, 微缺口 $m$ 附近选取不同场径时计算 得到的场强值及权函数见图 11。通过计算发现, 在材 料数个晶粒的尺寸上计算得到的微缺口处场强值明显 低于试样上施加的名义应力 (190 MPa), 这明显与实际 不符。而在取合适场强范围内 $\left(\sigma_{F I}>190 \mathrm{MPa}\right)$, 因场强 值随场径值的变化波动较大, 很难找到一个准确的 $\Omega$ 。

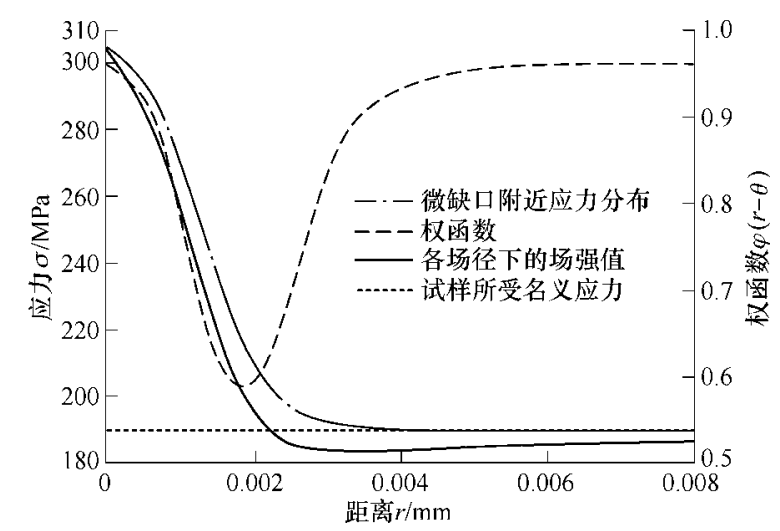

图 11 采用传统场强法分析微缺口 $m$ 附近应力场 (190 MPa)

通过分析，在分析微观小尺寸应力场时，传统场 强法之所以存在以上问题，其原因有：(1) 因为应力集 中的原因, 微缺口附近的应力梯度往往较常规缺口更 大, 场强值在此处随场径变化而产生的波动也比较大, 
而当应力变化平缓时, 往往已是应力变化远端, 其应力 大小趋近于构件上所施加的名义应力, 已不足以反应缺 口处的应力状态; (2) 对于权函数, 其大小主要取决于 参考点处的应力梯度与距缺口的距离, 且两者互为权 数。距离微缺口较近时, 因为应力梯度大且变化平稳, 因而距离参数对应的权数较大, 在权函数的影响因素 中起主导作用。在此区域内, 权函数的变化趋势与最 初假设相符, 随距离增大呈下降趋势, 但此范围的数 量级远远小于数个晶粒的尺寸, 甚至会小于单个晶粒 的尺寸(图 11), 传统场强法的基本假设在此已不适用。 当距离微缺口较远时, 因为应力梯度较大, 距离参数 整体较大且变化相对平稳, 因而此时鉴于微缺口处应 力分布特性应力梯度会急剧下降, 因而权函数数值会 相应的急剧增加。在此区域内, 权函数的变化趋势与 最初假设相反，也与传统场强法的基本假设是相悖的。

为解决本文提出的微缺口问题, 针对传统场强 法存在的不足，本文采用一种改进的场强法来计算 微缺口处的场强值, 其形式为

$$
\begin{gathered}
\sigma_{F I}=\sigma_{\max }-\frac{\int_{\Omega}\left(\sigma_{\max }-\sigma_{r-\theta}\right) \varphi(r-\theta) \mathrm{d} \nu}{\int_{\Omega} \varphi(r-\theta) \mathrm{d} v} \\
\varphi(r-\theta)=\left(r\left|\frac{\mathrm{d} \sigma_{r-\theta}}{\sigma_{\text {max }} \mathrm{d} r}\right| \cos \theta\right)^{\frac{r}{R}}
\end{gathered}
$$

同样以初始加载应力 $190 \mathrm{MPa}$ 下的板试样为 例, 采用改进场强法计算微缺口 $m$ 附近选取不同场 径时的场强值及权函数, 计算结果见图 12。计算发 现，改进方法计算得到的场强值更依赖于各点处的 应力梯度值, 其采用权函数间接定义了 $\Omega$, 在一定 程度上弱化了场径值对计算结果的影响, 因此改进 模型计算的结果更加稳定，同时也更接近真实值。

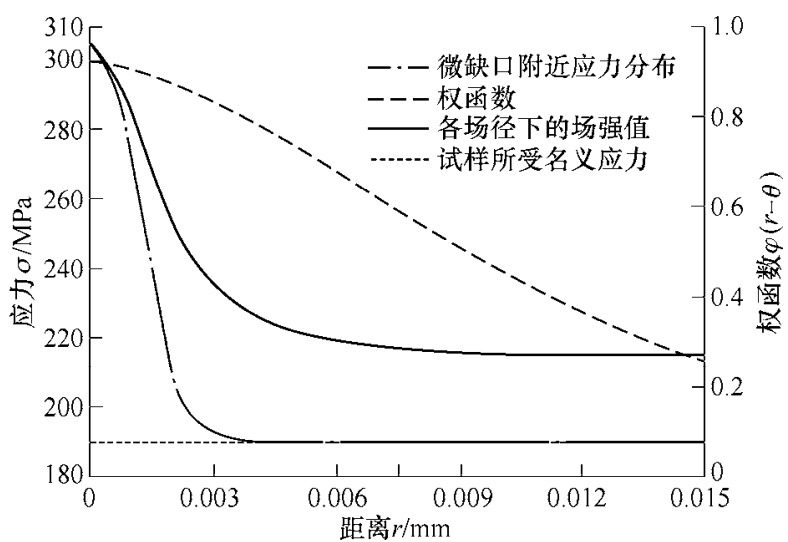

图 12 采用改进场强法分析微缺口 $m$ 附近应力场 (190 MPa)

为验证以上两个模型在本问题中的计算精度, 以 $50 \% 、 97.5 \%$ 两种典型可靠度为例, 分别采用以 上两个模型计算各组试样微缺口处有效应力, 并将 结果进行对比。首先通过以下公式计算八组试样在
$50 \% 、 97.5 \%$ 可靠度下的疲劳极限值

$$
\sigma_{p}=\sigma_{\mu}+\beta \mu_{p} \sigma_{\mathrm{s}}
$$

式中, $\sigma_{p}$ 为可靠度为 $p$ 下试样疲劳极限; $\sigma_{\mu}$ 为试 样疲劳极限均值; $\sigma_{\mathrm{s}}$ 为试样疲劳极限标准差; $\beta$ 为 与配对数有关的标准修正系数; $\mu_{p}$ 为可靠度 $p$ 对应 的标准正态偏量。采用式(7), 八组试样在 $50 \%$ 、 97.5\%可靠度下的疲劳极限值计算结果见表 3(其中 参数 $\beta 、 \mu_{p}$ 可查标准 HBZ112-1986 得到)。

表 3 各组试样在 $50 \% 、 97.5 \%$ 可靠度下的疲劳极限值

\begin{tabular}{ccc}
\hline \multirow{2}{*}{ 试样代号 } & \multicolumn{2}{c}{ 疲劳极限 } \\
\cline { 2 - 3 } & $p=50 \%$ & $p=97.5 \%$ \\
\hline A-1 & 179 & 159 \\
A-2 & 211 & 197 \\
A-3 & 218 & 197 \\
B-1 & 172 & 158 \\
B-2 & 206 & 171 \\
B-3 & 216 & 194 \\
C-1 & 187 & 165 \\
C-2 & 221 & 202 \\
\hline
\end{tabular}

针对以上八组试样对应的疲劳极限大小, 分别采 用本文的有限元模型求解试样面部、角部、曲面部微 缺口附近应力分布, 并分别通过原始场强法和改进场 强法求取试样危险部位的场强值。因场强法中定义的 场强值对应的是同种材料光滑试样的名义应力，因此 需将两种模型的计算结果与同材料理想状况下的疲 劳极限进行对比(厂家提供的材料理想条件下的疲劳 极限标称值: 50\%-225 MPa，97.5\%-200 MPa), 疲劳 极限计算结果及对比结果见表 4。值得注意的是，因 板试样面部与角部粗粘度有多种组合, 为确定每组试 样的有限元分析条件及有效应力计算位置, 在计算过 程中结合了每组试样的打磨方式(表 1)以及结果中面 断裂与角断裂出现的次数比例(表 4), 以此定义了各组 试样的分析计算条件(表 5, 其中有两个部位的表示取 两个部位计算结果的均值)。表中误差定义如下

$$
\mu=\frac{\sigma-\sigma_{p}}{\sigma_{p}} \times 100 \%
$$

式中, $\sigma$ 为施加与本组疲劳极限同等大小的名义应力 时求得的试样局部危险部位场强值(有效应力)大小。

表 4 各型板试样中面断裂占总疲劳失效类别的比例

\begin{tabular}{cc}
\hline 试样代号 & 面断裂占比 $(\%)$ \\
\hline A-1 & 0 \\
A-2 & 42.86 \\
A-3 & 20.00 \\
\hline B-1 & 22.22 \\
B-2 & 60.00 \\
B-3 & 28.57 \\
\hline
\end{tabular}


表 5 不同可靠度下两种方法疲劳极限计算结果对比

\begin{tabular}{|c|c|c|c|c|c|c|c|c|c|}
\hline \multirow{3}{*}{ 试样代号 } & \multirow{3}{*}{ 分析计算条件 } & \multicolumn{4}{|c|}{ 原场强法 } & \multicolumn{4}{|c|}{ 改进场强法 } \\
\hline & & \multicolumn{2}{|c|}{$p=50 \%$} & \multicolumn{2}{|c|}{$p=97.5 \%$} & \multicolumn{2}{|c|}{$p=50 \%$} & \multicolumn{2}{|c|}{$p=97.5 \%$} \\
\hline & & 计算值/MPa & 误差(\%) & 计算值/MPa & 误差(\%) & 计算值/MPa & 误差(\%) & 计算值/MPa & 误差(\%) \\
\hline A-1 & 角部( $R a=1.6)$ & 178 & -20.9 & 158 & -21.0 & 234 & -2.5 & 210 & 5.0 \\
\hline A-2 & $\begin{array}{l}\text { 角部 }(R a=0.4) \\
\text { 面部 }(R a=1.6)\end{array}$ & 209 & -7.1 & 195 & -2.5 & 231 & 2.7 & 218 & 9.0 \\
\hline A-3 & 角部 $(R a=0.4)$ & 217 & -3.6 & 195 & -2.5 & 224 & -0.4 & 203 & 1.5 \\
\hline B-1 & 角部 $(R a=1.6)$ & 171 & -24.0 & 157 & -21.5 & 226 & 0.4 & 209 & 4.5 \\
\hline B-2 & $\begin{array}{l}\text { 角部 }(R a=0.4) \\
\text { 面部 }(R a=1.6)\end{array}$ & 204 & -9.3 & 169 & -15.5 & 228 & 1.3 & 192 & -4.0 \\
\hline B-3 & 角部 $(R a=0.4)$ & 215 & -4.4 & 192 & -4.0 & 222 & -1.3 & 199 & -0.5 \\
\hline C-1 & 曲面部( $R a=1.6)$ & 187 & -16.9 & 165 & -17.5 & 228 & 1.3 & 203 & 1.5 \\
\hline $\mathrm{C}-2$ & 曲面部( $R a=0.4)$ & 221 & -1.8 & 202 & 1.0 & 227 & 0.9 & 208 & 4.0 \\
\hline
\end{tabular}

通过表 5 对比可以发现, 采用传统场强法计算 得到的结果误差波动大, 且所求场强值普遍远小于 同条件下的对应的疲劳极限标称值, 这与图 12 反映 的结果是一致的。采用改进场强法计算得到的结果 误差波动小, 且所求场强值普遍接近对应的标称值, 结果较传统场强法大为改观。因此, 改进方法对本 研究中的微缺口问题更为适用, 且计算结果也更为 合理。

\section{3 基于改进场强法的不同部位粗糙度分析}

在第 2.2 节分析的基础上, 采用改进场强法 分别计算两种试样在表 2 中各应力等级下不同 部位微缺口处的应力场强值, 并将分析结果绘制 于图 13。

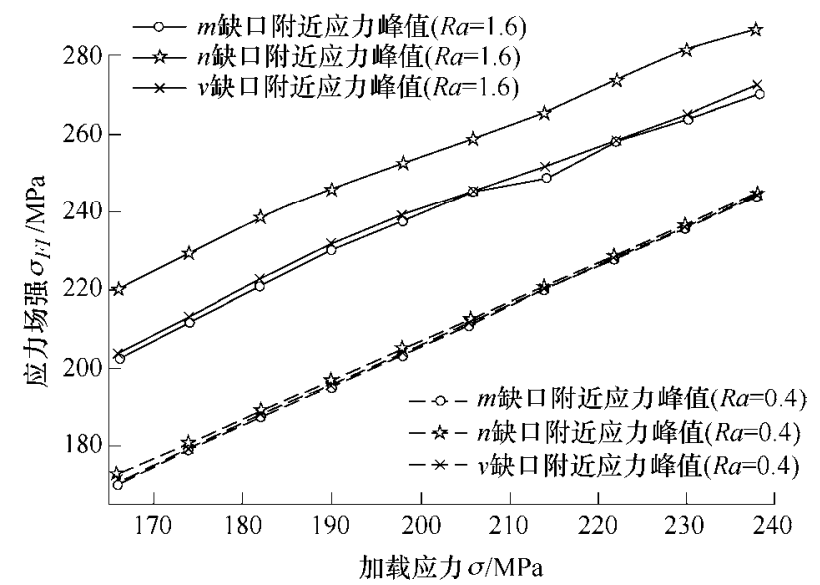

图 13 不同加载应力下微缺口附近应力场强值

为更为直接地反映不同部位粗糙度影响的差异 性, 定义有效应力集中系数 $K$

$$
K=\frac{\sigma_{F I}}{\sigma_{\max }}
$$

采用式(8), 计算各应力等级下的微缺口处有效 应力集中系数, 并将计算结果绘制到图 14 中。

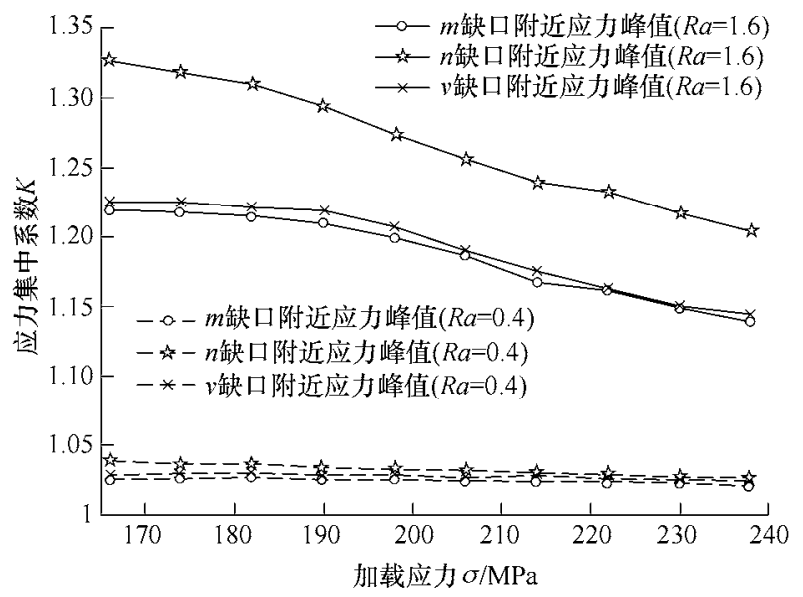

图 14 不同加载应力下微缺口附近的有效应力集中系数

通过总结图 13、14 可以发现，改进场强法的分 析结果与试验结果基本一致, 现通过以下两点进行 讨论分析。

\subsection{1 不同厚度板试样结果讨论分析}

(1) 通过试验结果图 4 和表 4 可以发现, 在试 样角部和面部保持同等表面质量的前提下，两类试 样在两种表面质量的情况下角断裂出现的比例远大 于面断裂, 见表 6 。同样观察计算结果图 14 可以发 现, 在粗糙度等级分别为 $0.4 、 1.6$ 时, 同样加载力 下角部 $(n$ 缺口)应力集中都明显大于面部。

\section{表 6 同样表面质量下角断裂、面断裂占比(试验结果)}

\begin{tabular}{ccc}
\hline 试样代号 & 角断裂占比(\%) & 面断裂占比(\%) \\
\hline A-1 & 100 & 0 \\
A-3 & 80.00 & 20.00 \\
\hline B-1 & 77.78 & 22.22 \\
B-3 & 71.43 & 28.57 \\
\hline 加权平均 & 82.76 & 17.24 \\
\hline
\end{tabular}

通过这一分析可以得出结论, 对于同等表面质 量的试样, 角部微缺口引起的试样表面应力集中现 象明显高于面部微缺口, 即同等打磨量的前提下, 试样角部比面部更易出现疲劳裂纹源。 
(2) 通过观察计算结果图 15 可以发现, 不同加 载力下, 面粗䊁度与角粗粘度对试样疲劳性能的影 响差异性是不同的。图 15 列出了不同加载力下粗粘 度等级分别为 $0.4 、 1.6$ 时面部微缺口和角部微缺口 处的应力集中相对差值, 相对差值的计算参照式 (9), 通过观察图 15 可以发现, 随着加载力的增大, 面部微缺口和角部微缺口处的应力集中相对差值也 随之不断增大。同样对比试验结果图 16 可以发现, 在其他条件都相同的条件下, 试样面断裂所处的应 力级总体高于角断裂所处的应力级, 这一现象主要 是由于面粗楉度与角粗粘度对试样疲劳性能的影响 差异大小造成的：两者差异性越大(即 $\mu_{K}$ 正向越 大), 角部应力集中越明显, 角部更易出现疲劳裂纹 源; 两者差异性越小(即 $\mu_{K}$ 正向接近 0 ), 面部应力 集中越接近角部, 面部出现疲劳裂纹源的概率增大。

$$
\mu_{K}=\frac{K_{J}-K_{M}}{K_{M}} \times 100 \%
$$

式中, $\mu_{K}$ 为面部微缺口和角部微缺口处的应力集中 相对差值; $K_{J}$ 为角部应力集中系数; $K_{M}$ 为面部应 力集中系数。

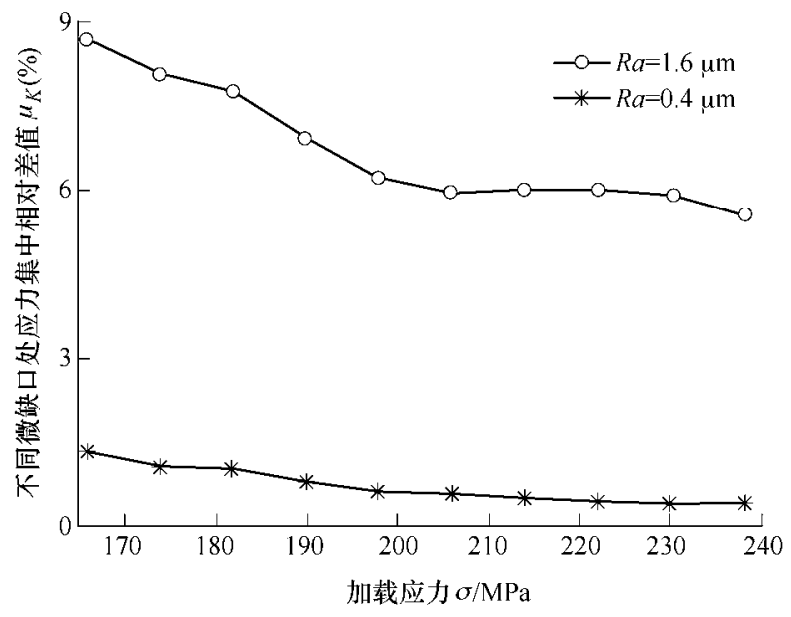

图 15 不同微缺口处应力集中相对差值 (计算结果)

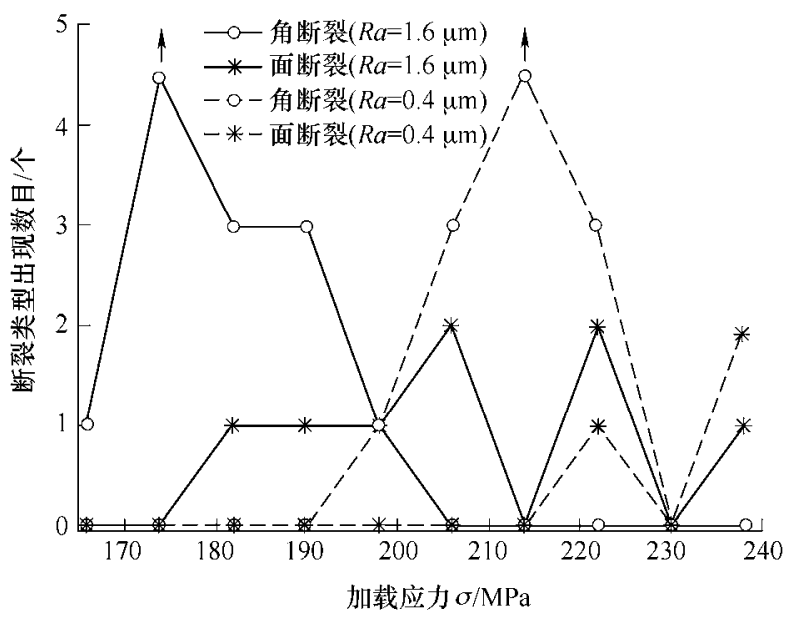

图 16 各应力等级下出现不同断裂类型的数目(试验结果)
以上通过对比同一粗糙度试样在不同加载力下的 试验及计算结果可以得出结论: 对于相同表面质量的试 样, 加载应力越高, 面部集中系数与角部集中系数越相 近, 即面粗粘度与角粗糙度对试样疲劳性能的影响差 异越小, 试样失效形式逐渐由角断裂向面断裂转化。

(3) 通过观察试验结果表 6 可以发现, 打磨过后 试样面断裂的占比会有所上升, 说明随着表面质量的 提高, 面部微缺口与角部微缺口影响的差异性有所减 弱。通过观察计算结果图 15 同样可以发现, 高表面 质量下所求面部微缺口和角部微缺口处的应力集中 相对差值明显小于低表面质量时的相对差值。总结可 得，在相同加载力下试样表面质量越高，面部微缺口 处集中系数与角部微缺口处集中系数越相近, 即面粗 䊁度与角粗䊁度对试样疲劳性能的影响差异越小。

\subsection{2 板试样与棒试样结果讨论分析}

对于棒材试样组结果的分析, 需结合板试样试 验结果进行对比, 对于本组的对比, 本研究更关心 的是几种典型部位粗粘度的影响差异性, 因此在此 暂时忽略试样厚度的影响, 将以上八组试验数据进 行重新配对, 分别得到两种粗粘度下面断裂、曲面 断裂、角断裂三种情形下的疲劳极限, 见图 17。对 于计算结果, 在图 14 的基础上分别计算两种粗䊅度 下曲面部微缺口与另外两种微缺口处的应力集中相 对差值, 见图 18。通过观察试验结果图 17、计算结 果图 18 可以发现, 存在于棒试样上的曲面部粗粘度 影响程度界于面部与角部之间, 且对于曲率为 $4 \mathrm{~mm}$ 的曲面, 其影响更接近于面部的影响。这一点可以 解释为, 对于不同部位粗䊁度影响的分析过程中, 任何曲率对应的曲面都处于面部到角部的过渡区， 即当曲面半径趋于无穷大时, 曲面部即可等效为面 部; 当曲率半径趋于无穷小时, 曲面部即可等效为 角部。在此基础上可进一步得出, 曲面部微缺口与 另外两种微缺口处的对应关系仍符合上小结 “不同 厚度板试样结果讨论” 中得到的三条结论。

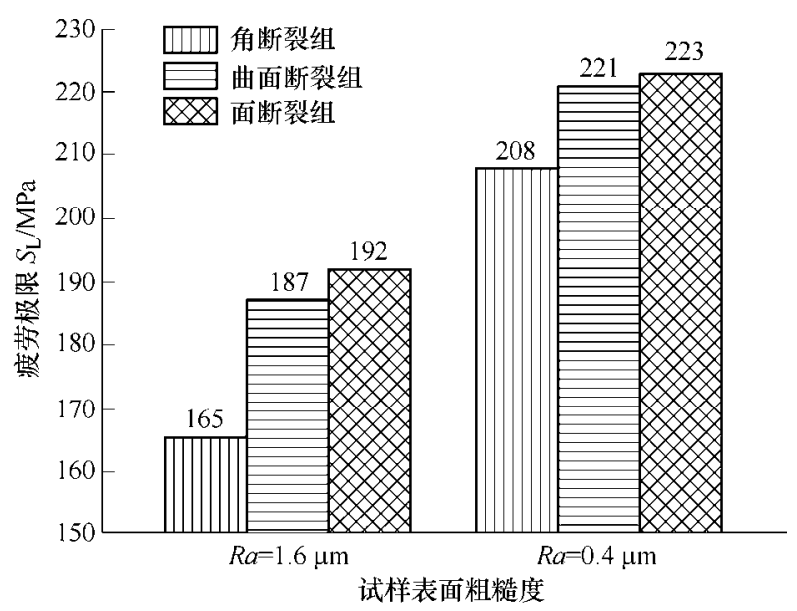

图 17 三种断裂形势下的疲劳极限统计结果(试验结果) 


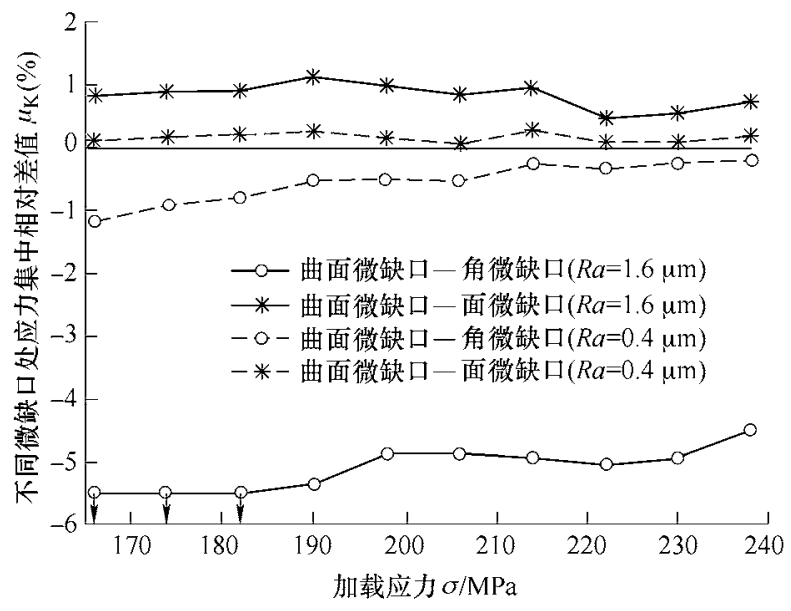

图 18 曲面部微缺口与另外两种微缺口处的应力集中相对 差值(计算结果)

值得注意的是, 对于只打磨角部的试样(角部 $R a=0.4 \mu \mathrm{m}$, 面部 $R a=1.6 \mu \mathrm{m})$, 理论分析结果与试验 结果略有差异。对于这组试样, 理论计算结果显示 因角部微缺口引起的有效应力集中系数明显低于面 部, 而图 4 试验结果中此组试样仍出现了大量的角 断裂现象。究其原因, 是因为本研究只在定义表面 粗糙度的量级上, 分析了试样表面微缺口引起的应 力集中现象。而对于试样疲劳寿命, 除了受这一参 数影响外, 还受裂纹扩展过程、材料夹杂物、表面 处理手段以及试样加工手段的影响, 而这些影响因 素大多优先推动角断裂的出现, 从而导致只打磨角 部的试样也出现了大量角断裂。同时, 结合试验过 程, 部分裂纹虽起源于试样面部, 但有时面部裂纹 源距角部距离较近, 在裂纹扩展过程中裂纹会优先 向角部扩展, 在最终断裂后这部分试样往往会被误 判为角断裂。另外, 疲劳试验结果本身就具有一定 的分散性, 例如, 对于未打磨试样(角部 $R a=1.6 \mu \mathrm{m}$, 面部 $R a=1.6 \mu \mathrm{m}$ ), 理论计算结果显示因表面微缺口 引起的角部有效应力集中系数明显高于面部, 而图 5 试验结果中未打磨的试样仍出现了少量的面断裂 现象。因此, 对于疲劳试验结果分析, 理论分析的 结果只能反映试验结果的整体趋势, 从这一角度来 讲, 即使对于只打磨角部的试样, 本节的理论分析 结果也仍是合理的。

\section{3 结论}

(1) 对于同等表面质量的试样, 角部微缺口引 起的试样表面应力集中现象明显高于面部微缺口, 即同等打磨量的前提下, 试样角部比面部更易出现 疲劳裂纹源。

(2) 对于相同表面质量的试样, 加载应力越高, 面部集中系数与角部集中系数越相近, 即面粗粘度
与角粗粘度对试样疲劳性能的影响差异越小, 试样 失效形式逐渐由角断裂向面断裂转化。

(3) 在相同加载力下试样表面质量越高, 面部 微缺口处集中系数与角部微缺口处集中系数越相 近, 即面粗糙度与角粗楉度对试样瘦劳性能的影响 差异越小。

（4）任何曲率对应的曲面处于面部到角部的过 渡区, 因此曲面部粗糙度影响程度界于面部与角部 之间, 且其与另外两种微缺口的对应关系仍符合以 上三条结论。

另外, 从实际工程应用的角度看, 本研究具有 很高的工程应用价值。依据传统理论, 对构件的表 面处理往往不会区分相对部位, 而且校核过程只分 析其面部粗鋉度, 以致造成构件面部表面质量冗 余过大, 或角部表面质量不能达到要求。本文中 的定量分析方法恰好弥补了传统理论的这一不 足, 能为工程构件的表面处理提供更为合理的指 导, 避免因试样表面处理不当而造成资源浪费甚 至引发工程事故。

\section{参 考 文 献}

[1] WANG Jinlong, ZHANG Yuanliang, SUN Qingchao, et al. Giga-fatigue life prediction of FV520B-I with surface roughness[J]. Materials \& Design, 2016, 89: 1028-1034.

[2] 宋波, 章媛洁, 赵晓, 等. 激光选区熔化增材与机加工 复合制造 AISI 420 不锈钢: 表面粗粘度与残余应力演 变规律研究 [J]. 机械工程学报, 2018, 54(13): 170-178. SONG Bo, ZHANG Yuanjie, ZHAO Xiao, et al. Selective Laser melting and subtractive hybrid manufacture AISI420 stainless steel: Evolution on surface roughness and residual stress[J]. Journal of Mechanical Engineering, 2018, 54(13): 170-178.

[3] PU Wei, ZHU Dong, WANG Jiaxu, et al. Rolling-sliding contact fatigue of surfaces with sinusoidal roughness[J]. International Journal of Fatigue, 2016, 90: 57-68.

[4] JONES J L, MCMURTREY M D, BROWN L B, et al. The effect of crack wake roughness on the environmentally assisted fatigue behavior of an aerospace Al-alloy in low moisture environments[J]. Engineering Fracture Mechanics, 2018, 199: 343-357.

[5] 刘军, 刘勇俊, 刘永寿, 等. 开孔试样的表面粗粘度对 疲劳寿命影响的定量分析[J]. 中国机械工程, 2008, 19(3): 327-329.

LIU Jun, LIU Yongjun, LIU Yongshou, et al. Quantitative analysis of roughness effect on fatigue life of specimen with open holes[J]. China Mechanical Engineering, 2008, 19(3): 327-329. 
[6] SILVA P S C P D, CAMPANELLI L C, CLAROS C A E, et al. Prediction of the surface finishing roughness effect on the fatigue resistance of Ti-6Al-4V ELI for implants applications[J]. International Journal of Fatigue, 2017, 103: 258-263.

[7] WANG Jinlong, ZHANG Yuanliang, ZHAO Qingchen, et al. The fatigue failure analysis and fatigue life prediction model of FV520B-I as a function of surface roughness in HCF regime[J]. Journal of Materials Research, 2017, 32(3): 634-643.

[8] HATAMLEH O, LYONS J, FORMAN R. Laser peening and shot peening effects on fatigue life and surface roughness of friction stir welded 7075-T7351 aluminum[J]. Fatigue \& Fracture of Engineering Materials \& Structures, 2010, 30(2): 115-130.

[9] BAGEHORN S, WEHR J, MAIER H J. Application of mechanical surface finishing processes for roughness reduction and fatigue improvement of additively manufactured Ti-6Al-4V parts[J]. International Journal of Fatigue, 2017, 102: 135-142.

[10] LI Changyou, DAI Weibing, DUAN Fei, et al. Fatigue life estimation of medium-carbon steel with different surface roughness[J]. Applied Sciences, 2017， 7(4): 338.

[11] LACERDA J C D, MARTINS G D, SIGNORETTI V T, et al. Corrigendum to "evolution of the surface roughness of a low carbon steel subjected to fatigue"[J]. International Journal of Fatigue, 2017, 102: 143-148.

[12] MEREUTA V, BUCIUMEANU M, PALAGHIAN L. 3D roughness parameters as factors in determining the evolution of effective stress concentration factors in fatigue processes[J]. Applied Mechanics \& Materials, 2013, 248(1): 504-510.

[13] KYAW S T, ROUSE J P, LU J, et al. Effects of surface roughness on thermo-mechanical fatigue life of a P91 power plant steel[J]. Procedia Structural Integrity, 2016, 2: 664-672.

[14] AMOR M B, BELGHITH S, MEZLINI S. Finite element modeling of RMS roughness effect on the contact stiffness of rough surfaces[J]. Tribology in Industry, 2016, 38(3): 392-401.

[15] ANDREWS S, SEHITOGLU H. A computer model for fatigue crack growth from rough surfaces[J]. International Journal of Fatigue， 2000， 22(7): 619-630.

[16] 廖智奇, 吴运新, 袁海洋. 表面粗糙度对三维应力集中 系数及疲劳寿命的影响 $[\mathrm{J}]$. 中国机械工程, 2015, 26(2): 147-151.

LIAO Zhiqi, WU Yunxin, YUAN Haiyang. Influences of surface roughness on three dimensional stress concentration factor and fatigue life[J]. China Mechanical
Engineering, 2015, 26(2): 147-151.

[17] MISRA A, PANDEY P M, DIXIT U S. Modeling and simulation of surface roughness in ultrasonic assisted magnetic abrasive finishing process[J]. International Journal of Mechanical Sciences, 2017， 133: 344-356.

[18] AROLA D, WILLIAMS C L. Estimating the fatigue stress concentration factor of machined surfaces[J]. International Journal of Fatigue, 2002, 24(9): 923-930.

[19] EMBRECHTS J J. The random-incidence scattering coefficient of infinite periodic surfaces with rectangular and sine-shaped roughness profiles[C/CD]// Forum Acusticum, Aalborg, Denmark. 2011.

[20] 程正坤, 廖日东, 李玉婷, 等. 表面形貌对应力集中系 数的影响研究 $[\mathrm{J}]$. 北京理工大学学报, 2016, 36(3): 231-236.

CHENG Zhengkun, LIAO Ridong, LI Yuting, et al. Effect of surface topography on stress concentration factor[J]. Transactions of Beijing Institute of Technology, 2016, 36(3): 231-236.

[21] SURARATCHAI M, LIMIDO J, MABRU C, et al. Modelling the influence of machined surface roughness on the fatigue life of aluminium alloy[J]. International Journal of Fatigue, 2008， 30(12): 2119-2126.

[22] ALANG N A, RAZAK N A, MISKAM A K. Effect of surface roughness on fatigue life of notched carbon steel[J]. International Journal of Engineering \& Technology, 2011, 11(1): 160-163.

[23] ALENCAR G, JESUS A M P D, RUI A B C, et al. Fatigue life evaluation of a composite steel-concrete roadway bridge through the hot-spot stress method considering progressive pavement deterioration[J]. Engineering Structures, 2018, 166: 46-61.

[24] GAUR V, ENOKI M, OKADA T, et al. A study on fatigue behavior of MIG-welded Al-Mg alloy with different filler-wire materials under mean stress[J]. International Journal of Fatigue, 2018, 10: 119-129.

[25] ZHAO Bingfeng, XIE Liyang, BAI Xin, et al. A multi-axial low-cycle fatigue life prediction model considering effects of additional hardening[J]. Fatigue \& Fracture of Engineering Materials \& Structures, 2018, 41(7): 1488-1503.

[26] YADOLLAHI A, MAHTABI M J, KHALILI A, et al. Fatigue life prediction of additively manufactured material: effects of surface roughness, defect size, and shape[J]. Fatigue \& Fracture of Engineering Materials \& Structures, 2018, 41(7): 1602-1614.

[27] HAGHSHENAS A , KHONSARI M M. Damage accumulation and crack initiation detection based on the evolution of surface roughness parameters[J]. 
International Journal of Fatigue, 2018, 107: 130-144.

[28] BIAN Xinxiao, LI Wei, QIU Lifang. Study on C-R-S curves of up-and-down test and fatigue limit stress[J]. Journal of University of Science \& Technology Beijing, 2010, 32(3): 380-383.

[29] BAI Xin , XIE Liyang, ZHANG Ruijin, et al. Measurement and estimation of probabilistic fatigue limits using Monte-Carlo simulations[J]. International Journal of Fatigue, 2017, 95: 229-235.

[30] 尚德广, 王大康, 李明, 等. 随机疲劳寿命预测的局部 应力应变场强法[J]. 机械工程学报, 2002, 38(1): 67-70. SHANG Deguang, WANG Dakang, LI Ming, et al. Method of local stress-strain fieid intensity for random fatigue life prediction[J]. Chinese Journal of Mechanical Engineering, 2002, 38(1): 67-70.

[31] YAO Weixing, YE Bin, ZHENG Lichun. A verification of the assumption of anti-fatigue design[J]. International Journal of Fatigue, 2001, 23(3): 271-277.

[32] LIU Gang, TANG Liulun, HUANG Yi. A study on the influence factors of fatigue in welded joints based on field inten-sity approach[J]. Journal of Ship Mechanics, 2014, 18(1): 158-164.

[33] WANG Mingchun, SONG Enpeng, ZHAO Qinghua. The prediction of mutiaxial high cycle fatigue based on stress field in-tensity[J]. Aircraft Design, 2015, 35(4): 10-13.

作者简介: 赵丙峰(通信作者), 男, 1991 年出生, 博士研究生。主要研 究方向为结构疲劳可靠性。

E-mail: 15940488242@163.com

谢里阳, 男, 1962 年出生, 博士, 教授, 博士研究生导师。主要研究方 向为大型工业系统风险概率分析与安全管理决策、结构强度、完整性与 设备状态诊断与监测和计算机仿真、虚拟试验与计算机辅助工程分析 (CAE)。

E-mail: lyxie@mail.neu.edu.cn 